\title{
Relationship between the level of glycosylated hemoglobin and diabetic retinopathy
}

\author{
Mingyan Yu ${ }^{1, a}$, Bin Song', Yonghua Xuan ${ }^{1}$, Hong Xu${ }^{1}$ \\ ${ }^{1}$ Binzhou Vocational College, Shandong, 256600, China \\ ${ }^{2}$ Affiliated Hospital of Binzhou Medical College, Shandong, 256600, China \\ aemail:yumingyan1977@163.com
}

Keywords: Glycosylated hemoglobin; Diabetic retinopathy; Type 2 diabetes mellitus

\begin{abstract}
To investigate the relationship between glycosylated hemoglobin (HbA1c) level and diabetic retinopathy (DR), we selected 260 patients with type 2 diabetes mellitus in Affiliated Hospital of Binzhou Medical College from January to December in 2016.These patients were examined by HbA1c and other biochemical indexes, fundus examination and fundus fluorescein angiography analysis. Experiments show that compared with the diabetic group with no obvious retinopathy, there was a high level of glycosylated hemoglobin in both the non proliferative DR Group and the proliferative DR Group, and the difference was statistically significant $(\mathrm{p}<0.05)$. So we can draw a conclusion that HbA1c level is positively correlated with DR patients, and HbA1c assay can be an important index for monitoring DR.
\end{abstract}

\section{Introduction}

Diabetes mellitus is a common disease of endocrine system. It is caused by a variety of causes of chronic hyperglycemia characterized by metabolic diseases, which can affect the whole body organs[1]. According to a survey only about One-fourth of people with diabetes can control their blood sugar at normal or near normal levels[2]. With the course of diabetes gradually prolonged, patients often appear hypertension, neurological lesions, ocular lesions and other complications. Diabetes can cause a lot of eye diseases.The common ocular abnormalities are abnormal lens metabolism, iris ciliary inflammation, glaucoma, diabetic retinopathy (DR) and so on. DR is one of the most important complications of diabetes mellitus, and is one of the major eye diseases affecting human vision health and even blindness in the world [3].

At present, the treatment of DR is mainly laser, but laser treatment can only delay. The disease can not be cured. Therefore, in order to minimize the damage of DR to the eyesight of patients with diabetes mellitus, it is very important to find out the abnormal fundus so as to make early diagnosis and effective treatment in time. Glycosylated hemoglobin (HbA1c) is a product of early glycosylation.The American Diabetes Association has identified HbA1c as the core standard for monitoring blood glucose levels in diabetics, which can be used as a monitoring indicator for the recent 4-12 weeks of diabetic blood glucose control and as an important indicator for predicting diabetic microvascular complications.

In this paper we are to investigate the correlation between the degree of diabetic retinopathy and glycosylated hemoglobin (HbA1c), and the results are reported as follows.

\section{Design of the Experiment}

\subsection{Information and Methods}

\subsubsection{Subjects}

We selected 260 cases of diabetes in Affiliated Hospital of Binzhou Medical College from January to December in 2016. There are 134 men and 126 women in the cases. The ages of these cases are from 25 to 82 years old.The average age is (67 \pm 10$)$ years old.The Courses of these 
patients are from 3 months to 25 years. The average course is $(6.74 \pm 4.56)$ years.

\subsubsection{Test Index}

The patients were fasting for the early morning fasting venous blood $4 \mathrm{ml}$, and the upper serum was isolated in 30min. The blood samples were detected by automatic biochemical analyzer.

The test indexes include fasting blood glucose (FPG), glycosylated hemoglobin (HbA1c), triglyceride (TG), total cholesterol (TC), low-density lipoprotein cholesterol (LDL-C), high-density lipoprotein cholesterol (HDL-C).

\subsubsection{Methods}

The same ophthalmologist of Affiliated Hospital of Binzhou Medical College examined the patient's fundus with a fundus lens after dilation. The results of fundus examination were divided into the group without retinopathy and the group with retinopathy. For patients with retinopathy, in order to determine the degree of retinal microvascular lesions for staging patients, professional ophthalmologists shall conduct fluorescence angiography of the retina. Combined with the results of fundus examination and retinal fluorescence angiography, the subjects were divided into three groups. The first group is non - diabetic retinopathy group(NDR).The second group is the DR group of nonproliferativediabetic retinopathy group(NPDR).The third group is the DR group of proliferativediabetic retinopathy group(PDR). There were 97 people in the NDR group, accounting for $37.31 \%$. There were 109 people in NPDR, accounting for 41.54\%. A total of 54 people in the PDR group, accounting for $20.77 \%$.

\subsubsection{Statistical processing}

Statistical software SPSS 17.0 was used to statistically process the relevant data of each group, and the data of all research indicators were expressed as mean value plus or minus standard deviation 2.

\section{Test results}

\subsection{Comparison of general data between the three groups.}

Compared with the simple diabetes group, the duration of disease in the non-proliferative DR group and the proliferative DR group was longer.The difference was statistically significant $(\mathrm{P}<$ 0.05). While the difference in age, gender, body mass index, systolic and diastolic blood pressure was not statistically significant $(\mathrm{P}>0.05)$. Specific results are shown in table 1.

\subsection{Comparison of glycosylated hemoglobin between the three groups.}

Compared with the diabetic group without obvious retinopathy, the levels of glycosylated hemoglobin were high in the non-proliferative DR group and the proliferative DR group, and the difference was statistically significant $(\mathrm{P}<0.05)$. The specific results are shown in table 2.

\subsection{Comparison of other biochemical indicators between the three groups.}

Compared with the simple diabetes group,the levels of FPG, TG and SCr in the non-proliferative DR group and the hyperplastic DR group were high, and the difference was statistically significant ( $\mathrm{P}<0.05)$, while the differences of TC and BUN in the three groups were not statistically significant $(\mathrm{P}>0.05)$. The specific results are shown in table 3.

\subsection{Comparison of clinical indicators between the three groups.}

Compared with the simple diabetes group,the visual acuity and corrected visual acuity decreased significantly in the non-proliferative DR group and the proliferative DR group $(\mathrm{P}<0.05)$, while the difference was statistically significant $(\mathrm{P}<0.05)$. The specific data analysis results are shown in table 4. 
Table 1 Comparison of three groups of general information

\begin{tabular}{|c|c|c|c|c|}
\hline & $\begin{array}{c}\text { Item simple } \\
\text { diabetes group } \\
(\mathrm{n}=97)\end{array}$ & $\begin{array}{c}\text { Non-proliferative } \\
\text { DR group } \\
(\mathrm{n}=109)\end{array}$ & $\begin{array}{c}\text { The proliferative } \\
\text { DR group } \\
(\mathrm{n}=54)\end{array}$ & $\mathrm{P}$ \\
\hline Age & $53.65 \pm 6.24$ & $54.34 \pm 7.08$ & $54.78 \pm 7.79$ & 0.7763 \\
\hline Sex(M/F) & $53 / 44$ & $59 / 50$ & $33 / 22$ & 0.8532 \\
\hline Course (Y) & $4.6 \pm 4.2$ & $9.7 \pm 5.8$ & $14.6 \pm 5.2$ & 0.0899 \\
\hline Index & $18.9 \pm 3.8$ & $19.3 \pm 4.1$ & $19.7 \pm 3.1$ & 0.7965 \\
\hline SBP & $131.39 \pm 12.62$ & $154.25 \pm 10.44$ & $162.16 \pm 11.53$ & 0.6432 \\
\hline DBP & $84.03 \pm 7.92$ & $95.23 \pm 8.15$ & $98.16 \pm 7.35$ & 0.7421 \\
\hline
\end{tabular}

Table 2 Comparison of the three groups of HbA1c

\begin{tabular}{|c|c|c|c|c|}
\hline & $\begin{array}{c}\text { Item simple } \\
\text { diabetes group } \\
(\mathrm{n}=97)\end{array}$ & $\begin{array}{c}\text { Non-proliferative } \\
\text { DR group } \\
(\mathrm{n}=109)\end{array}$ & $\begin{array}{c}\text { The proliferative } \\
\text { DR group } \\
(\mathrm{n}=54)\end{array}$ & $\mathrm{P}$ \\
\hline $\mathrm{HAb} 1 \mathrm{c}(\%)$ & $8.92 \pm 2.31$ & $11.36 \pm 3.42$ & $13.86 \pm 5.18$ & 0.0166 \\
\hline
\end{tabular}

Table 3 Comparison of other biochemical indexes between the three groups

\begin{tabular}{|c|c|c|c|c|}
\hline & $\begin{array}{c}\text { Item simple } \\
\text { diabetes group } \\
(\mathrm{n}=97)\end{array}$ & $\begin{array}{c}\text { Non-proliferative } \\
\text { DR group } \\
(\mathrm{n}=109)\end{array}$ & $\begin{array}{c}\text { The proliferative } \\
\text { DR group } \\
(\mathrm{n}=54)\end{array}$ & $\mathrm{P}$ \\
\hline FPG $(\mathrm{mM})$ & $9.40 \pm 2.51$ & $12.98 \pm 3.53$ & $14.32 \pm 4.68$ & $0.0227^{\mathbf{\Lambda}}$ \\
\hline TG $(\mathrm{mM})$ & $1.74 \pm 0.86$ & $3.57 \pm 1.88$ & $4.65 \pm 1.25$ & $0.0076^{\mathbf{\Lambda}}$ \\
\hline TC $(\mathrm{mM})$ & $4.60 \pm 1.08$ & $4.65 \pm 1.33$ & $4.73 \pm 1.10$ & 0.7761 \\
\hline $\mathrm{BUN}(\mathrm{mM})$ & $4.65 \pm 1.52$ & $4.85 \pm 1.26$ & $5.02 \pm 2.83$ & 0.1233 \\
\hline $\mathrm{SCr}(\mu \mathrm{M})$ & $81.42 \pm 40.11$ & $101.06 \pm 35.89$ & $121.11 \pm 64.45$ & $0.0166^{\mathbf{\Lambda}}$ \\
\hline
\end{tabular}

Table 4 comparison of clinical indicators between the three groups

\begin{tabular}{|c|c|c|c|c|}
\hline & $\begin{array}{c}\text { Item simple } \\
\text { diabetes group } \\
(\mathrm{n}=97)\end{array}$ & $\begin{array}{c}\text { Non-proliferative } \\
\text { DR group } \\
(\mathrm{n}=109)\end{array}$ & $\begin{array}{c}\text { The proliferative } \\
\text { DR group } \\
(\mathrm{n}=54)\end{array}$ & $\mathrm{P}$ \\
\hline Vision & $0.65 \pm 1.08$ & $0.36 \pm 1.33$ & $0.13 \pm 1.10$ & $0.0191^{\mathbf{\Lambda}}$ \\
\hline $\begin{array}{c}\text { Correction } \\
\text { vision }\end{array}$ & $0.75 \pm 1.04$ & $0.24 \pm 1.01$ & $0.19 \pm 0.99$ & $0.0216^{\mathbf{\Lambda}}$ \\
\hline $\begin{array}{c}\text { Intraocular } \\
\text { pressure }\end{array}$ & $16.03 \pm 0.75$ & $18.00 \pm 0.83$ & $19.07 \pm 0.68$ & 0.8580 \\
\hline
\end{tabular}

\section{Conclusion}

Based on the analysis of the experiment results, we can conclude that the higher the level of HbA1c, the faster the occurrence and progress of DR. And abnormal HbA1c level is an important risk factor for poor vision in DR patients, and with the gradual increase of HbA1c value, the visual acuity of patients presents a significant downward trend.

\section{Discussion}

Diabetes is a chronic metabolic disease that causes complications in all parts of the body. One of 
the most common microvascular complications is diabetic retinopathy [4] [5]. Clinical data have shown that the occurrence and progression of DR is related to the level of hba1c. HbA1c is the product of the combination of blood glucose and hemoglobin, and the blood glucose is constantly fluctuating. Each examination can only reflect the blood glucose level at that time, while the glycosylated hemoglobin is gradually generated. Therefore, compared with blood glucose, its level is quite stable and not easy to decompose. The amount of glycosylated hemoglobin can reflect the long-term blood glucose level in the body and the total blood glucose concentration of the patients in the last 8 to 12 weeks [6]. Therefore, the detection of glycosylated hemoglobin in diabetic patients is more valuable than blood glucose detection, and is more conducive to the detection of diabetes control conditions and early detection of diabetic retinopathy[7] [8] [9].

The results of this study show that abnormal HbA1c level is an important risk factor for poor vision of DR patients, and with the gradual increase of HbA1c value, the visual acuity of patients presents a significant downward trend.

In this study, it was also found that the incidence of blood lipids, creatinine and urea nitrogen abnormalities in the non-proliferative DR group and the proliferative DR group was significantly higher and more complex than that in the normal fundus group, suggesting that with the extension of the disease course of diabetic patients, DR and other systemic complications were gradually emerging [10]. Moreover, the higher the level of hba1c, the faster the occurrence and progress of DR, and the more complicated and serious the systemic complications. This means that effective treatment of all kinds of complications should be carried out in the clinical treatment of diabetes.

In conclusion, the visual condition of DR patients will seriously affect the quality of life of the patients, and it has interaction with HbA1c. Therefore, for diabetics with a disease course of more than 5 years, routine HbA1c test and regular fundus examination should be carried out at an early stage to detect DR early and treat it early, so as to effectively avoid the risk of blindness of diabetic patients.

\section{Acknowledgement}

In this paper, the research was sponsored by the Nature Science Foundation of Binzhou City (Project No. 2014ZC0122).

\section{References}

[1] Shen Ying, Chen Ling, Lu Lei-qun. Analysis on related factors ofretinopathy in type 2 diabetes [J]. Shanghai Medicine, 2009, 32(12):1120-1121

[2] Mazhar K,Varma R,Choudhury $\mathrm{F}$ et al.Severity of diabetic retinopathy and health-related quality of life:the Los Angeles Latino Eye Study[J].Ophthalmology,2011,118:649-655

[3] Bamashmus M A,Gunaid A A,Khandekar R B.Diabetic retinopathy,visual impairment and ocular status among patients with diabetes mellitus in Yemen: a hospitalbased study[J] Indian Ophthalmol, 2009,57( 4) : 293- 298

[4] Li Jin-feng. Discussion on the relationship between diabetic retinopa-thy and related risk factors [J]. Guide of China Medicine, 2009, 17(7):115-116.

[5] Su Li. Analysis of the relationship between glycated hemoglobin and diabetes and its complications [J]. Journal of clinical and Experimental Medicine, 2011, 10(10): 771-773

[6] Jiang guo-yan, zhang huifang. Practical diabetes science. People's health press, 1992: 63-64.

[7] Shen ying, Chen ling, lu lei qun. Analysis of related factors in type 2 diabetic retinopathy [J]. Shanghai medical science, 2009, 32(12): 1120-1121

[8] Massin P,Lange C,Tichet J,et al.Hemoglobin A1c and fasting plasmaglucose levels as predictors of retinopathy at 10 years:the French DESIR study[J].Arch Ophthalmol,2011,129:188-195. 
[9] Ma J,Wang X,Wang Y,et al.The relationship between glycatedhemoglobin and complexity of coronary artery lesions among olderpatients with diabetes mellitus[J].PLoS One,2014,9 (3) :e91972.

[10] Reith S,Battermann S,Hellmich M,et al.Impact of type 2 diabetesmellitus and glucose control on fractional flow reserve measurementsin intermediate grade coronary lesions[J].Clin Res Cardiol,2014,103(3): 191-201. 